\title{
Perirhinal Cortex mGlu5 Receptor Activation Reduces Relapse to Methamphetamine Seeking by Restoring Novelty Salience
}

\author{
Jamie Peters', Michael D Scofield', Shannon M Ghee', Jasper A Heinsbroek' and Carmela M Reichel, \\ 'Department of Neurosciences, Medical University of South Carolina, Charleston, SC, USA
}

Rats that have self-administered methamphetamine (meth) under long access, but not short access, conditions do not recognize novel objects. The perirhinal cortex is critical for novelty detection, and perirhinal metabotropic glutamate 5 receptors (mGlu5) are downregulated after long-access meth. The novel positive allosteric modulator (PAM) I-(4-(2,4-difluorophenyl) piperazin-I-yl)-2-((4fluorobenzyl)oxy)-ethanone, or DPFE, demonstrates improved solubility compared with other mGlu5 PAMs, thus allowing brain-sitespecific pharmacological studies. Infusion of DPFE into perirhinal cortex restored novel object recognition in long-access meth rats. To investigate the impact of these cognitive enhancing effects on relapse, we tested the effects of DPFE infusions into perirhinal cortex on meth-seeking under two different test conditions. In the standard cue relapse test, perirhinal DPFE infusions did not alter meth-seeking in the presence of meth cues. However, in a novel cue relapse test, wherein animals were allowed to allocate responding between a novel cue and meth-conditioned cue, perirhinal DPFE infusions shifted the pattern of responding in long-access rats toward a profile resembling short-access rats, which respond equally for novel and meth cues. Perirhinal mGlu5 are thus a promising pharmacological target for the restoration of cognitive function in meth addicts. Targeting these receptors may also reduce relapse, particularly in situations where novel stimuli compete with conditioned stimuli for control over meth seeking.

Neuropsychopharmacology (2016) 4I, |477-|485; doi:I0.1038/npp.2015.283; published online 2I October 20I5

\section{INTRODUCTION}

Methamphetamine (meth) abuse is a growing problem in the United States and worldwide. Cognitive deficits have been observed in meth users, and these deficits are greater after extended meth use (Dean et al, 2013; Marshall and O'Dell, 2012). In human studies, it is difficult to infer whether such cognitive deficits are a cause or consequence of meth use; thus, animal models are useful in determining the directionality of this relationship. Self-administration models are currently the gold standard for studying addiction in rats, and in recent years, models that employ longer, extended access to drug have received growing attention, owing to their greater face validity and the noted differences between long- and short-access models (Ahmed and Koob, 1998; Rogers et al, 2008). Long access, but not short access, meth leads to a highly reproducible escalation of meth intake that is thought to reflect a more 'addicted' state compared with short-access models, which may reflect 'recreational' drug use (Ahmed and Koob, 1998).

Long-access meth leads to deficits in novel object recognition, a basic cognitive function that engages a neural

\footnotetext{
*Correspondence: Dr CM Reichel, Department of Neurosciences, Medical University of South Carolina, 173 Ashley Avenue, Charleston, SC 29425, USA, Tel: +I 843792 2487, Fax: +I 843792 4423, E-mail: reichel@musc.edu

Received 27 April 2015; revised 17 August 2015; accepted 26 August 2015; accepted article preview online 14 September 2015
}

circuit comprising the prefrontal cortex, hippocampus, and the perirhinal cortex, but only the latter is critical for novelty detection (Kealy and Commins, 2011; Warburton and Brown, 2014). Long-access meth results in a number of neuroadaptations within the perirhinal cortex that may underlie this cognitive deficit, including a downregulation of glutamate receptors, specifically GluN2B-containing $\mathrm{N}$-methyl-D-aspartate (NMDA) receptors and metabotropic glutamate 5 receptors (mGlu5; Reichel et al, 2014; Reichel et al, 2011; Scofield et al, 2015), both of which have been implicated in novel object recognition (Barker et al, 2006a; Barker et al, 2006b). Furthermore, restoring activity at perirhinal NMDA receptors using the partial agonist D-cycloserine restores novel object recognition in long-access meth rats (Scofield et al, 2015).

Systemic administration of the mGlu5-positive allosteric modulator (PAM) 3-cyano-N-(1,3-diphenyl-1H-pyrazol-5-yl) benzamide, or CDPPB, similarly restores novel object recognition in long-access meth rats (Reichel et al, 2011). With the recent advent of a more soluble, selective mGlu5 PAM, 1-(4-(2,4-difluorophenyl) piperazin-1-yl)-2-((4-fluorobenzyl)oxy)- ethanone, or DPFE (Gregory et al, 2013), a potential perirhinal locus for this effect can now be investigated using brain-site specific microinfusions. The goal of Experiment 1 was to determine whether intraperirhinal infusions of DPFE can restore novel object recognition in long-access meth rats, with the basic hypothesis that restoring glutamate receptor transmission in perirhinal cortex may be a viable strategy for restoring 
novel object recognition. Conversely, blocking glutamate transmission in this brain region disrupts novel object recognition in rodents and primates (Barker et al, 2006b; Malkova et al, 2015).

In order to determine whether meth-induced impairments in novelty detection have any bearing on relapse vulnerability, we set out to develop a model of relapse in Experiment 2, wherein novel cues compete with methconditioned cues. We hypothesized that such a model would engage the perirhinal cortex, which to date has not been implicated in relapse, despite its extensive interconnectivity with other brain regions, including the prefrontal cortex, nucleus accumbens, and amygdala (Kealy and Commins, 2011), all known to mediate relapse (Nawata et al, 2012; Rocha and Kalivas, 2010). Given that short-access meth rats do not exhibit deficits in novelty recognition (Reichel et al, 2012a; Rogers et al, 2008), and animals have an innate propensity to explore and interact with novel objects (Ennaceur and Delacour, 1988), we hypothesized that a novel cue would invigorate responding as well as a meth cue in short-access rats. In long-access rats, however, we anticipated that their deficits in novelty detection would bias them toward greater responding for the meth cue.

Finally, Experiment 3 was designed to examine the role of perirhinal mGlu5 in this new model of relapse, which we refer to here as a novel cue relapse test. This would not only implicate perirhinal cortex in relapse involving competition between novel and meth cues, but also identify a receptor system that could be therapeutically targeted to restore novelty salience and reduce relapse. Furthermore, this would imply that similar neural mechanisms regulate novel object recognition and novel cue detection in an operant model of relapse, thus underscoring the importance of a common meth-induced deficit in novelty salience.

\section{MATERIALS AND METHODS}

\section{Subjects}

A total of 57 male Sprague-Dawley rats (Harlan) weighing 250-275 g on arrival were individually housed on a reversed 12 : 12 light-dark cycle (1800 hours light on); all experiments were conducted during the dark cycle. Food (standard rat chow, Harlan, Indianapolis, IN) was restricted to $20 \mathrm{~g}$ chow/ day during experimentation; water was available ad libitum. Procedures were conducted in accordance with the 'Guide for the Care and Use of Laboratory Rats' (Institute of Laboratory Animal Resources on Life Sciences, National Research Council) and approved by the IACUC of the Medical University of South Carolina.

\section{Surgery}

Rats were anesthetized with ketamine $(57 \mathrm{mg} / \mathrm{kg}$, i.p.), xylazine ( $8.7 \mathrm{mg} / \mathrm{kg}$, i.p.), and Equithesin ( $0.7 \mathrm{ml} / \mathrm{kg}$, i.p.). Ketorolac (2.0 mg/kg, i.p.) and cefazolin (200 mg/kg, s.c.) were administered preoperatively for analgesia and to protect against post-surgical infection, respectively. Intravenous catheters were surgically implanted as previously described (Reichel et al, 2014). For experiments requiring intracranial microinfusions, guide cannulas (Plastics One, Roanoke, VA) directed at the perirhinal cortex were stereotaxically implanted after the catheter surgery, under the same plane of anesthesia. Perirhinal coordinates were measured from bregma at the skull surface, angled $10^{\circ}$ laterally, as follows: anterior-posterior (AP) $-4.8 \mathrm{~mm}$, medial-lateral (ML) $-5 \mathrm{~mm}$, DV $-7.5 \mathrm{~mm}$. When necessary, an anesthesia booster of ketamine $(\sim 1 / 6$ th of initial dose) was administered to maintain sedation throughout the stereotaxic surgery. Rats were allowed to recover 5-10 days before initiating behavioral procedures.

\section{Drugs and Microinfusions}

Meth (Sigma, St Louis, MO) was dissolved in sterile saline. The novel mGlu5-positive allosteric modulator 1-(4- $(2,4-$ difluorophenyl)piperazin-1-yl)-2-((4-fluorobenzyl)oxy) ethanone (DPFE) was used to activate perirhinal mGlu5. DPFE was dissolved in a $20 \%$ solution of hydroxyl-propyl betacyclodextrin in distilled water at a concentration of $1 \mathrm{mg} / \mathrm{ml}$. This vehicle is sufficient to dissolve DPFE with gentle sonication to a point where it can pass through a microinjection needle, which is not possible with the more commonly used mGlu5 PAM CDPPB. The DPFE dose for intracranial studies $(0.5 \mu \mathrm{g} / 0.5 \mu \mathrm{l} /$ side $)$ was inferred from a preliminary study we performed with systemic dosing (Supplementary Experiment S1). Microinjectors extended $1.5-\mathrm{mm}$ beyond the cannula; bilateral microinfusions occurred simultaneously over $2 \mathrm{~min}$; injectors were left in place an additional minute to allow diffusion.

\section{Meth Self-Administration}

Self-administration was conducted in behavioral chambers $(30 \times 20 \times 20 \mathrm{~cm}$, Med Associates, St Albans, VT) as previously described (Reichel et al, 2014). Responding on the active lever delivered a 2-s infusion of meth $(20 \mu \mathrm{g}$ in $50 \mu \mathrm{l})$ followed by an unsignaled $20 \mathrm{~s}$ timeout period (FI-20 s) where responding was without consequence (to prevent overdose). Positioned above the active lever was a white stimulus light used to signal each meth infusion. A speaker positioned near the ceiling of the chamber was used to deliver a tone $(78 \mathrm{~dB}, 4.5 \mathrm{kHz})$ in conjunction with the stimulus light for Experiment 1. Responding on the inactive lever never produced any consequence. The first week consisted of 1-h sessions. After seven sessions of acquisition, rats either continued for an additional 14 sessions of $1 \mathrm{~h}$ (short access), or they increased to $6 \mathrm{~h}$ (long access) sessions over the last 2 weeks of self-administration. Rats were then returned to their home cage for 1-2 weeks of abstinence prior to relapse testing and/or novel object recognition testing (see Experimental Procedures for details). Four rats were eliminated from the short-access group (Experiment 2) due to failure to meet acquisition criteria of at least 15 infusions on average over the last 3 days of meth selfadministration.

\section{Cue Relapse Testing}

Cue relapse testing was conducted by returning rats to their self-administration chambers after a period of home cage abstinence (eg no prior extinction training was conducted), and a single non-contingent cue presentation signaled the start of the test. Rats were not tethered and meth was not 
delivered during this 1 -h test. Responding on the active lever resulted in presentation of the light-tone stimulus $(5 \mathrm{~s}$ duration; FI-20 s) previously paired with meth during selfadministration.

\section{Novel Cue Relapse Testing}

For novel cue relapse tests, a novel lever and stimulus light, neither of which was present during self-administration, were positioned on the opposite wall from the active lever, to minimize association between the novel and meth cues at test. For this same reason, no tone was used during selfadministration or testing. The novel stimulus light was covered with pieces of Velcro on the top and bottom to provide a tactile stimulus and reduce light intensity (compared with the meth cue) by leaving only a small slit for the light to disperse. Responding on the novel and active/ meth levers turned on their respective cue lights $(15 \mathrm{~s}$ duration; FI-20 s), but meth was not delivered. Responding on all levers was recorded over the $1 \mathrm{~h}$ session. Testing occurred after a period of home cage abstinence, and no prior extinction training was conducted. Data were converted to a Relapse Index to reflect the within-subject choice for the meth cue+lever $v s$ the novel cue+lever. The formula parallels that used to calculate the recognition index for novel object recognition:

Relapse index $=\frac{\text { Active lever presses }}{\text { Active lever presses }+ \text { novel lever presses }}$

Thus, a relapse index above 0.50 indicates higher responding for the meth cue $v s$ the novel cue, whereas an index of 0.50 indicates indifference for these cues. Results were also examined during the first 15-min bin of the session separately, since novelty is short lived, and time-course analyses revealed this was an optimal time point for detecting discrimination between novel and active levers. Figure $3 \mathrm{~b}$ depicts the layout of the operant chamber at test.

\section{Novel Object Recognition}

Novel object recognition testing was performed as previously described (Reichel et al, 2012a). On abstinence day 7 or 14 (see Experimental Procedures), rats explored two identical objects for $3 \mathrm{~min}$. Immediately after this familiarization session, vehicle or DPFE was administered. Testing was conducted $90 \mathrm{~min}$ later by allowing rats to explore an object from the familiarization phase and a novel object for $3 \mathrm{~min}$. Exploration time (seconds spent with novel or familiar object) was measured manually by an experimenter blind to treatment group using accelerated video playback with an event recorder in Ethovision XT 8.0 (Noldus, Leesburg, VA) and was defined as time spent actively sniffing or touching the object. Using these values the recognition index is calculated by dividing the time spent with the novel object by the time spent with both objects. Approaches to the object were also quantified, and an approach score was calculated (approaches to novel object over total approaches). Total distance $(\mathrm{cm})$ traveled was simultaneously recorded as a measure of ambulation during the test. Objects included a PVC pipe $(6.4 \times 3.8 \mathrm{~cm})$, a light bulb $(8.9 \mathrm{~cm})$, and/or a plastic bottle (Reichel et al, 2014). Objects and the arena were wiped down with $70 \%$ ethanol between animals. Animals were excluded from all analyses if they failed to sample each object for at least $3 \mathrm{~s}$ during familiarization $(n=2$ from Experiment 1), or if they failed to sample both objects during testing, or had less than $3 \mathrm{~s}$ total exploration time for both objects ( $n=2$ from Experiment 1$)$.

\section{Experimental Procedures}

Experiment 1: Restoring novel object recognition in longaccess meth rats by activating perirhinal mGlu5.

Rats $(n=16)$ underwent long access meth selfadministration procedures. For this experiment, each meth infusion during self-administration was paired with a 5-s compound light-tone stimulus in which the white stimulus light above the lever was delivered simultaneously with a tone. On abstinence day 7, rats were tested for novel object recognition. Vehicle or DPFE was microinfused into the perirhinal cortex immediately after object familiarization. After novel object recognition testing and an additional week of home-cage abstinence, rats underwent cue relapse testing on abstinence day 14. Vehicle or DPFE was microinfused into perirhinal cortex $15 \mathrm{~min}$ prior to this test. Group assignments were maintained between tests in case there were any lasting effects of the initial DPFE dosing, as tolerance has been noted with repeated administration of other mGlu5 PAMs (Parmentier-Batteur et al, 2010).

Experiment 2: Developing a novel cue relapse test to assess the impact of meth-induced cognitive deficits on relapse.

Rats $(n=29)$ were implanted with intravenous catheters and, after recovery from surgery, went through either long$(n=8)$ or short-access $(n=12)$ meth self-administration. Meth infusions were signaled by a 15 -s cue light above the active lever (eg the meth cue). On abstinence day 7, rats were returned to the behavioral chambers for a novel cue-relapse test (described in detail above). Just prior to testing, a subset of short-access rats $(n=9)$ received a priming injection of meth $(1 \mathrm{mg} / \mathrm{kg}$, i.p.), which has previously been shown to induce relapse in traditional extinction-reinstatement models (Reichel and See, 2010; Rocha and Kalivas, 2010). Thus, we reasoned that a meth prime should increase the relapse index in our novel cue relapse test. One rat in the meth prime group was a statistical outlier ( $>2 \mathrm{SD}$ from the mean) and was excluded from all analyses.

Experiment 3: Reducing relapse in a novel cue relapse test by activating perirhinal mGlu5.

Rats $(n=16)$ were implanted with an intravenous catheter and intracranial cannula directed at the perirhinal cortex. After recovery from surgery, they underwent long-access meth self-administration procedures. As in Experiment 2, a 15-s cue light above the active lever was used as the meth cue. On abstinence day 7, rats received an infusion of vehicle or DPFE into perirhinal cortex 15 min prior to placement in the chambers for novel cue relapse testing. The following week, rats underwent novel object testing on abstinence day 14 . Conditions for novel object testing were analogous to those described for Experiment 1.

\section{Statistical Analyses and Behavioral Observations}

Self-administration and relapse test data were analyzed using a one- or two-way analysis of variance (ANOVA) 
with post hoc as specified in the Results. Dunnett's post hoc was used when comparing with a single control group or time point, and Tukey's post hoc was used when making multiple comparisons across groups. Rats were assigned to vehicle or DPFE treatment groups in a balanced manner based on total meth intake, and two-way ANOVAs confirmed no group differences in self-administration responding (all $p$ 's $>0.05$ ). Relapse and recognition indices were analyzed using either a two-sample unpaired $t$-test comparing vehicle with DPFE treatment groups, or by oneway ANOVA (Experiment 2 relapse indices). In addition, for each treatment group, the relapse and recognition indices were compared with a hypothetical mean of 0.50 using twosample $t$-test. All statistical analyses were conducted with the alpha set at 0.05 , and all data are graphed as the mean \pm SEM.

\section{RESULTS}

Experiment 1: Restoring novel object recognition in longaccess meth rats by activating perirhinal mGlu5.

Consistent with our previous work (Reichel et al, 2011), we observed a robust escalation in meth intake as long-access progressed $(\mathrm{F}(20,315)=4.41, p<0.001$; Figure 1a). Total meth intake over the entire self-administration phase was on average $92 \pm 6 \mathrm{mg} / \mathrm{kg}$ (vehicle group $=98 \pm 10 \mathrm{mg} / \mathrm{kg}$; DPFE group $=85 \pm 6 \mathrm{mg} / \mathrm{kg}$ ). On abstinence day 7 , rats were tested for novel object recognition. The groups did not differ during familiarization (Supplementary Table S1A). Vehicle or DPFE was infused into perirhinal cortex after familiarization. Testing occurred $90 \mathrm{~min}$ later. The vehicle group performed at chance levels, whereas the DPFE group had a recognition index significantly above chance (eg $>0.50$ ) $(t(7)=4.59, p=0.003)$ and significantly greater than vehicletreated rats $(t(14)=2.24, p=0.042)$. DPFE did not cause any changes in ambulation during the test (Veh: $3190 \pm 147 \mathrm{~cm}$; DPFE: $3093 \pm 226 \mathrm{~cm}$ ). Furthermore, DPFE did not alter novel object recognition in saline controls (Supplementary Experiment S2).

As DPFE was able to restore novel object recognition in long-access meth rats, we tested whether this cognitive enhancement might also reduce relapse in the presence of meth cues. On abstinence day 14, rats were returned to the operant chambers for a cue relapse test, following perirhinal infusions of vehicle or DPFE. Responding on the active lever was higher than the inactive lever (main effect of lever $(\mathrm{F}(1,28)=47.90, p<0.001)$; Figure $2 \mathrm{a})$. No other effects were observed indicating that intraperirhinal DPFE does not alter meth-seeking under these conditions. Microinfusions were located predominantly at the entorhinal-perirhinal border, just ventral to the rhinal fissure (ie area 35, Figure $2 b$ ).

Experiment 2: Developing a novel cue relapse test to assess the impact of meth-induced cognitive deficits on relapse.

We hypothesized that the therapeutic effects of perirhinalapplied DPFE might be contingent on the presence of novel stimuli. Thus, we developed a self-administration/relapse model that incorporates choice between a novel cue and a meth-conditioned cue on the relapse test. Long-access meth rats escalated their meth intake (One way ANOVA over long-access Days 8 through $21,(\mathrm{~F}(7,111)=3.458, p<0.001)$; Figure $3 \mathrm{a})$, but short-access rats did not. Total meth intake in the long-access group $(117 \pm 11 \mathrm{mg} / \mathrm{kg})$ differed significantly from that in the short-access group $(18 \pm 2 \mathrm{mg} / \mathrm{kg} ; t(18)=$ 10.91, $p<0.001)$.

During the novel cue relapse test (see Figures $3 \mathrm{~b}$ for chamber layout), long- and short-access rats exhibited distinct profiles of responding over the three available levers (Figure 3c). A two-way ANOVA (lever $\times$ group) revealed a main effect of lever $(\mathrm{F}(2,54)=19.11, p<0.001)$ and a significant interaction $(\mathrm{F}(2,54)=7.7, p=0.001)$, but no main effect of long- $v s$ short-access. Tukey's post hoc comparisons revealed that in long-access rats, responding on the active lever was significantly higher than responding on the novel lever $(p<0.001)$ as well as the inactive lever $(p<0.001)$, but responding on the novel lever was not substantially higher than responding on the inactive lever. By contrast, in short-access rats, responding on the active lever $(p<0.05)$ and the novel lever $(p<0.01)$ were both significantly higher than responding on the inactive lever, but responding on the active lever was not substantially higher than responding on the novel lever. The groups did not differ in inactive lever responding, which is used as a measure of non-specific activity. Thus, the relapse index was calculated using only active and novel lever responding a

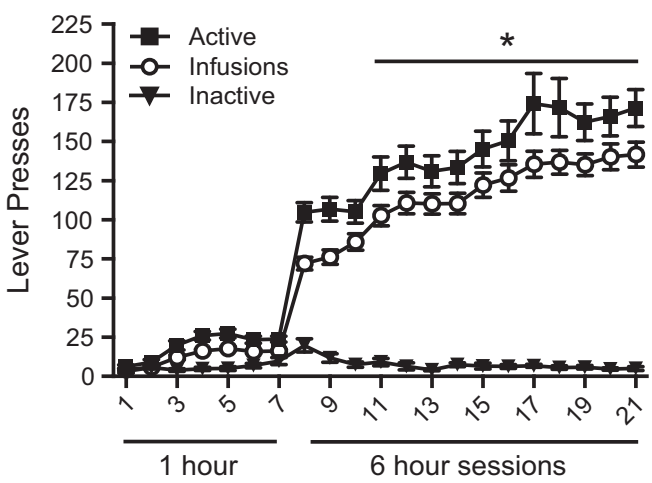

b

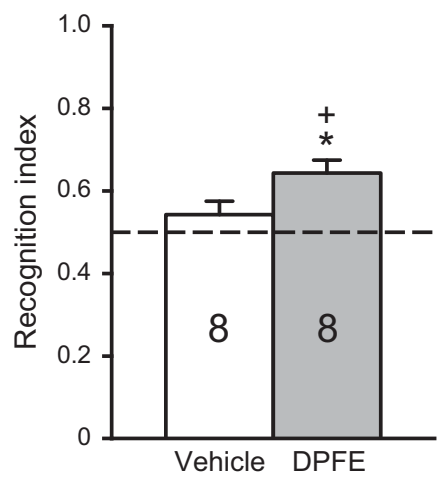

Figure I Long-access meth self-administration leads to escalated meth intake and impairments in novel object recognition that can be restored by intraperirhinal DPFE. (a) Self-administration behavior from Experiment I. ${ }^{*} p<0.05$ compared with the first long-access session (infusions). (b) Novel object recognition testing from Experiment I. $* p<0.05$ compared with a hypothetical mean of $0.50 .+p<0.05$ compared with vehicle. 
a

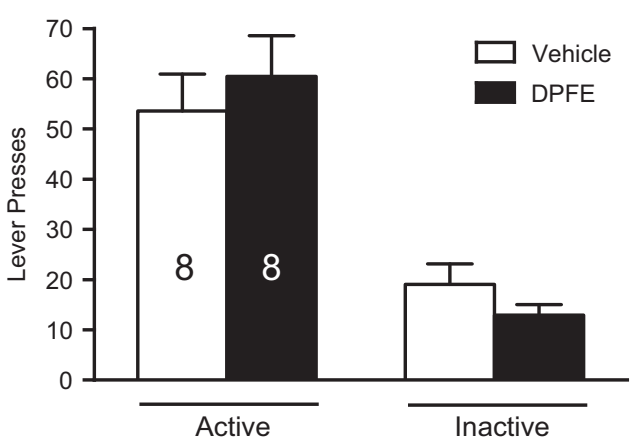

b

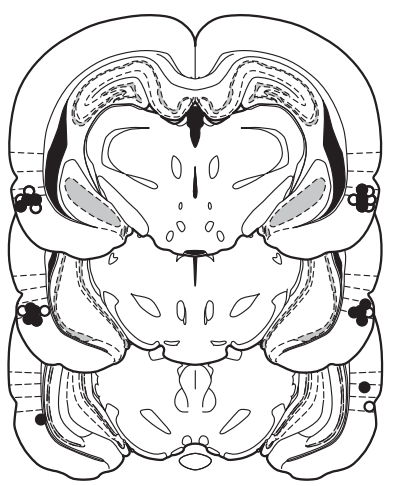

Figure 2 Relapse in the presence of meth-conditioned cues is not altered by intraperirhinal DPFE. (a) Total lever presses over the I-h cue relapse test from Experiment I. (b) Histological verification of microinjector needle placements within perirhinal cortex from Experiment I. White dots reflect the needle tip location for vehicle infusions; black dots reflect DPFE infusions. Placements were predominantly clustered in area 35, just below the rhinal fissure.

a

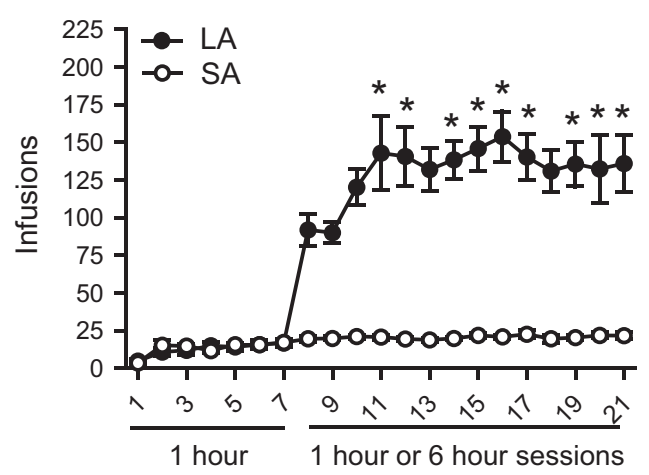

C

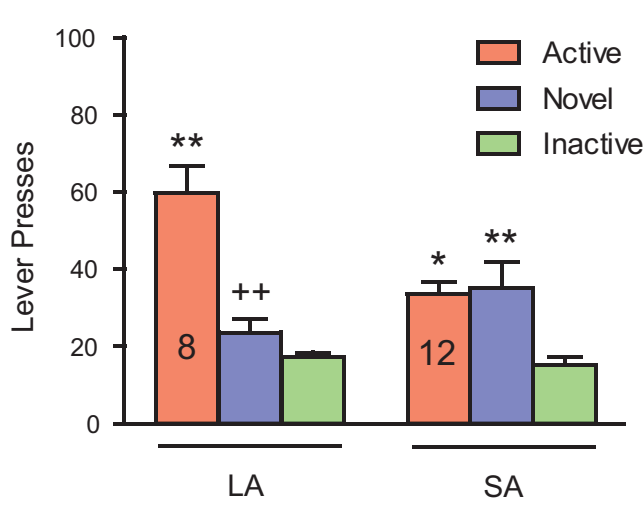

b

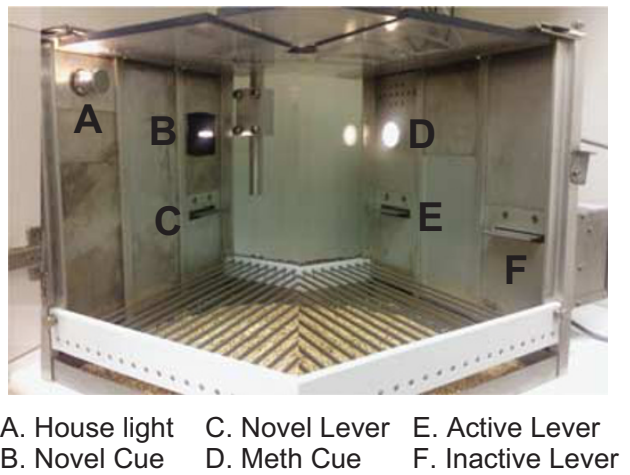

d

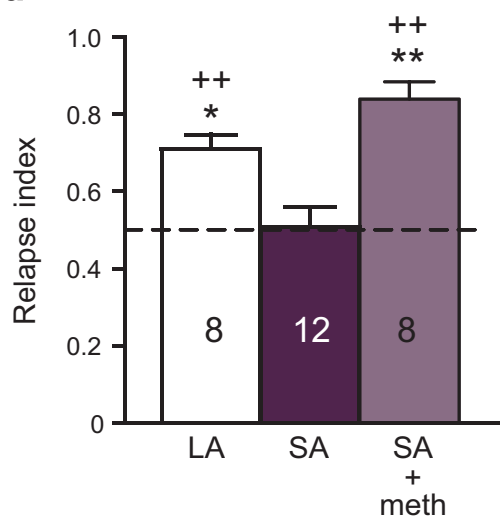

Figure 3 Short-access meth rats respond equally for novel and meth-conditioned cues in a relapse setting, but long-access meth rats respond preferentially for meth cues. (a) Long-access (LA) meth self-administration results in higher amounts of meth intake (and escalation) compared with short-access (SA) meth. ${ }^{*} p<0.05$ compared with the first long-access session. (b) Layout of the test chamber for Experiment 2. (c) Total lever presses over the I-h novel cue relapse test from Experiment 2. $* 0<0.05$, ** $p<0.01$ compared with inactive responding within access condition. $++p<0.0$ I compared with active responding within access condition. (d) Relapse index reflects within-subject choice between meth-cue vs novel-cue (see Materials and Methods for formula). $* p<0.05$, ** $p<0.0$ I compared with SA group. $++p<0.0$ I compared with hypothetical mean of 0.50 .

(active lever presses/total presses on both active and novel levers).

Short-access rats did not show a significant relapse index (eg above 0.50), whereas long-access rats did $(\mathrm{t}(7)=5.51$, $p<0.001$; Figure 3d), indicating they responded more for meth cues. In a subset of short-access rats, we administered a priming injection of meth $(1 \mathrm{mg} / \mathrm{kg}$, i.p.) just prior to the novel cue test. This resulted in a significant relapse index $(t(7)=7.48, p<0.001)$, similar to that in long-access rats (Figure 3d). Thus, it is possible to shift the profile of responding in short-access rats to one resembling longaccess rats. A one-way ANOVA $(\mathrm{F}(2,25)=12.69, p<0.001)$ 
a

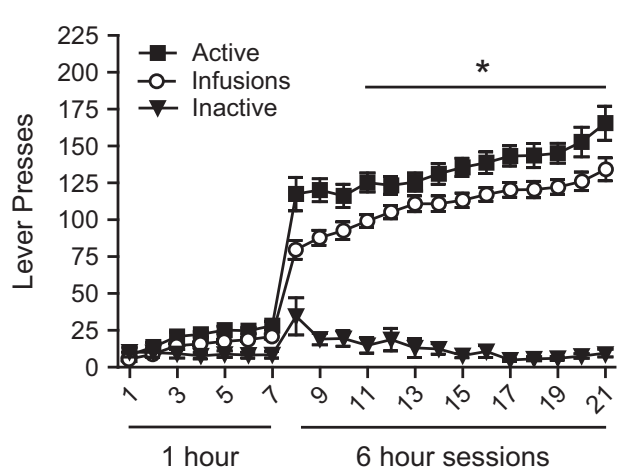

b

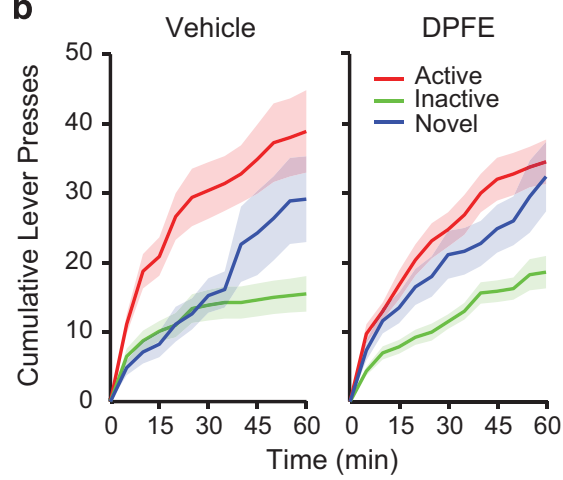

C

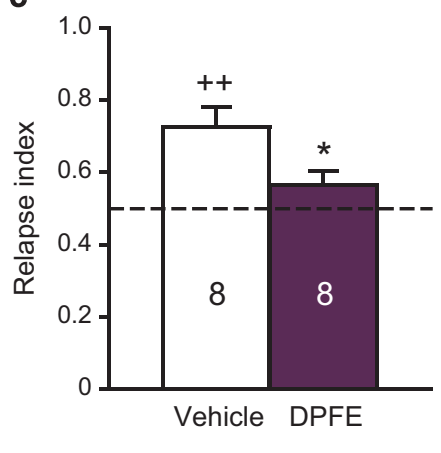

Figure 4 Intraperirhinal DPFE reduces relapse index in long-access meth rats subjected to the novel cue relapse test. (a) Self-administration behavior from Experiment 3. $* 0$ < 0.05 compared with the first long-access session (infusions). (b) Time course of lever pressing behavior over the I-h novel cue relapse test indicating more pronounced effects of treatment early in the session ( I5-min, eg before habituation to the novel cue). (c) Intraperirhinal DPFE reduces relapse index on the novel cue relapse test. ${ }^{*} p<0.05$ compared with vehicle. $++p<0.0$ I compared with a hypothetical mean of 0.50 .
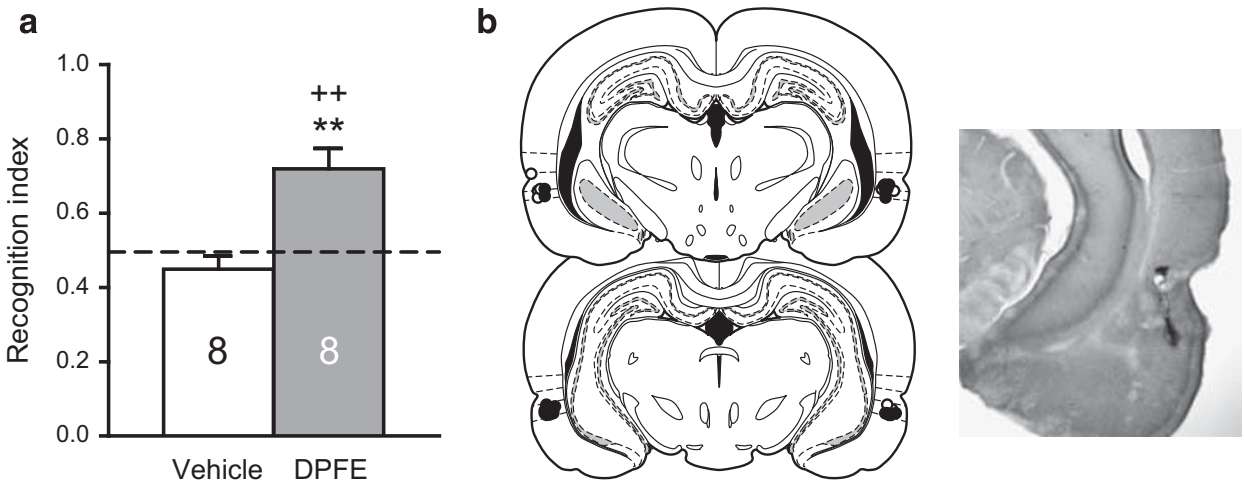

Figure 5 Intraperirhinal DPFE restored novel object recognition in the same cohort of animals tested in the novel cue relapse test. (a) Novel object recognition testing from Experiment 3. $* * 0<0.0$ I compared with vehicle; $++p<0.0$ I compared with a hypothetical mean of 0.50 . (b) Histological verification of microinjector needle placements within perishinal cortex from Experiment 3. The distribution of vehicle placements are indicated by white dots; black dots indicate DPFE placements. Infusions were predominantly clustered in area 35, just below the rhinal fissure. A section from a representative animal is shown at right.

revealed that both long-access rats (Dunnett's post hoc $p<0.05)$ and short-access rats receiving a meth prime (Dunnett's post hoc $p<0.001$ ) had a significantly higher relapse index than short-access rats not receiving a meth prime (Figure $3 \mathrm{~d}$ ).

Experiment 3: Reducing relapse in a novel cue relapse test by activating perirhinal mGlu5.

Given that it was possible to shift responding in shortaccess rats toward a pathological profile resembling longaccess rats by administering a meth prime, we next set out to reverse the pathological profile in long-access rats with perirhinal-applied DPFE. Long-access rats again exhibited an escalation in meth intake $(\mathrm{F}(13,195)=16.66, p<0.001$; Figure 4a). Total meth intake was on average $90 \pm 3 \mathrm{mg} / \mathrm{kg}$ (vehicle group $=89 \pm 5 \mathrm{mg} / \mathrm{kg}$; DPFE group $=90 \pm 6 \mathrm{mg} / \mathrm{kg}$ ). On abstinence day 7 , rats were returned to the operant chambers for novel cue relapse testing, following perirhinal infusions of vehicle or DPFE. A time-course analysis of responding over the 1-h test session indicated that differences between the groups were most pronounced early in the session (Figure 4b), when novelty is strongest (eg before habituation occurs). Thus, we restricted our analysis to the first $15 \mathrm{~min}$ of the session. Importantly, retrospective analysis of this time point did not alter effects from Experiments 1 and 2 (Supplementary Figure S3). Whereas the vehicle group exhibited a significant relapse index $(t(7)=4.419, p=0.004)$, the DPFE group did not $(p=0.1455$; Figure $4 c)$. Furthermore, the vehicle group had a significantly higher relapse index than the DPFE group $(t(14)=2.387, p=0.032)$, indicating that perirhinal-applied DPFE reduced relapse in this model.

After an additional week of home cage abstinence, on abstinence day 14, rats were tested for novel object recognition to reassess whether intraperirhinal DPFE produced similar therapeutic effects on the recognition index. As in Experiment 1, group assignments were maintained between tests. During the familiarization phase, the groups showed equivalent approach scores and time spent exploring each object (Supplementary Table S1B). Consistent with results from Experiment 1, the DPFE group showed significant novel object recognition $[\mathrm{t}(7)=3.996, p=0.005]$, whereas the vehicle group did not (Figure 5a). Furthermore, DPFE- 
infused rats performed significantly better than their vehicle counterparts $[\mathrm{t}(14)=4.134, p=0.001]$. DPFE did not alter general ambulation during the test (Veh: $2794 \pm 130 \mathrm{~cm}$; DPFE: $2943 \pm 108 \mathrm{~cm}$ ). Microinfusions were again located predominantly in area 35 (Figure 5b).

\section{DISCUSSION}

The present study identifies the therapeutic efficacy of activating perirhinal mGlu5 to both restore novelty recognition and reduce meth relapse by enhancing novelty salience. This new relapse model has several implications for meth relapse: (1) Novel stimuli can compete with methconditioned stimuli for control over meth-seeking behavior, (2) Prolonged or extended use of meth results in a loss of novelty salience, and (3) Restoring activity at perirhinal mGlu5 can restore novelty salience and reduce relapse. Presumably the latter compensates for previously observed meth-induced reductions of mGlu5 expression in perirhinal cortex (Reichel et al, 2011). These results underscore the importance of the perirhinal cortex, not just as a novelty detector, but also as a key component of the neural circuit controlling meth relapse, particularly under conditions where meth-conditioned cues compete with novelty for control over behavior.

\section{mGlu5, Novelty and Meth Addiction}

Antagonists of mGlu5 administered prior to, or in conjunction with, repeated meth injections protect against methinduced toxicity (Battaglia et al, 2002; Golembiowska et al, 2003). Thus, during initial drug exposure, mGlu5 antagonists might be protective. However, most addicts seek treatment only after they begin their attempt to abstain from drug use. Given that mGlu5 mediate some of the reinforcing properties of meth (Gass et al, 2008; Osborne and Olive, 2008), the downregulation in perirhinal mGlu5 observed after 7-14 days of abstinence (Reichel et al, 2011) may be a compensatory neuroadaptation to temper overactivation of these receptors during meth exposure. During abstinence, this adaptation could have negative consequences for neural processes, such as novelty detection, that rely on mGlu5. Thus, the treatment approach during abstinence may need to be different than the approach during drug exposure.

The mGlu5 PAM CDPPB has been shown to accelerate reductions in meth-seeking during extinction training (Kufahl et al, 2012). Furthermore, mGlu5 knockout mice exhibit delayed extinction of meth-seeking and increased cue-induced reinstatement (Chesworth et al, 2013). Chesworth et al, (2013) hypothesized that mGlu5 may have a role in mediating contextual salience, as both of these studies employed contextual cue extinction. Indeed, when extinction was conducted in an alternate context, the former group failed to observe an extinction-enhancing effect of CDPPB (Widholm et al, 2011). In contrast with the increased reinstatement reported by Chesworth et al, (2013) in the mGlu5 knockout, acute pharmacological antagonism of mGlu5 reduces cue-induced reinstatement of meth-seeking (Gass et al, 2008; Watterson et al, 2012). Developmental adaptations resulting from embryonic deletion of mGlu5 in the knockout may account for this apparent discrepancy.
Like long-access meth rats, transgenic mice expressing microRNA hairpins against mGlu5 mRNA under the control of a dopamine D1-receptor promoter exhibit deficits in novel object recognition (Parkitna et al, 2013). Similar to mGlu5 knockout mice (Olsen et al, 2010), these D1-mGlu5 knockdowns exhibit lower levels of operant sensation seeking, indicated by their reduced propensity to respond for novel cue lights (Parkitna et al, 2013). Interestingly, though they consume normal amounts of alcohol, they do not escalate their intake after a period of abstinence, whereas control mice typically double their intake (Parkitna et al, 2013). This phenotype suggests that mGlu5 are required for novel cue lights to engage responding, as well as for escalation to occur. This is consistent with our hypothesis that while activation of mGlu5 during initial meth exposure may promote methtaking, a downregulation in mGlu5 during meth withdrawal may lead to impairments in novelty salience. In addition, these findings underscore an important connection between mGlu5 and dopamine signaling.

\section{Novelty Detection vs Novelty Reward}

Although the observed meth-induced impairments in novel object recognition are generally interpreted as a deficit in novelty detection, these effects might also be explained by a deficit in novelty-induced reward. Nucleus accumbens dopamine release has been implicated in novelty choice behavior and indeed is a key substrate of novelty-induced place preference (Pierce et al, 1990; Rebec et al, 1997). Interestingly, novelty reward in a place preference model has been proposed as a measure of anhedonia (Bevins and Besheer, 2005). This is particularly relevant given that anhedonia has been reported in psychostimulant abusers after protracted withdrawal (Leventhal et al, 2008), and persistent decreases in striatal metabolism are proposed to underlie this anhedonic state (Wang et al, 2004). Although the long-access regimen of meth self-administration employed in the present study is not expected to induce striatal toxicity per se (Reichel et al, 2012b), there may be more subtle, detrimental effects on midbrain dopamine neuron physiology that lead to diminished novelty-induced dopamine release at nucleus accumbens terminals.

Perhaps another indication of the importance of dopamine in novel cue choice behavior is the observation that a priming injection of meth was able to shift responding away from the novel cue and toward the meth cue in short-access rats (Figure 3d). In the absence of this dopaminergic challenge, short-access meth rats respond equally for the novel $v s$ meth cue on the novel cue relapse test. This finding is consistent with a study by Bastle et al., (2012), who reported that short-access $(2 \mathrm{~h}$ daily self-administration sessions for 2 weeks) cocaine rats respond equally for novel and cocaine cues on a reinstatement test conducted after two weeks of extinction training. In the latter study, however, the novel and cocaine cues were tested in separate animals, so this model did not address the ability of novel cues to compete with conditioned drug cues in a choice setting. Although previous studies have demonstrated the ability of novelty (eg in a place preference model) to compete with the conditioned rewarding effects of cocaine (Reichel and Bevins, 2008,2010), to our knowledge this is the first to do so in an operant model of relapse. That a meth prime was able 
to shift behavior in short-access rats to a profile resembling long-access rats suggests that dopaminergic mechanisms, in addition to the perirhinal mGlu5 mechanism we identified here, mediate novel choice behavior in this paradigm. Future studies are needed to address the brain sites whereby this occurs and the potential interaction between dopamine and mGlu5 signaling.

Deficits in novel object recognition, or variants of the task, have been observed after repeated exposure to not only meth (Belcher et al, 2007; Gonzalez et al, 2014; O'Dell et al, 2010; Reichel et al, 2014; Reichel et al, 2012b; Rogers et al, 2008), but also cocaine (Briand et al, 2008), ecstasy (Schenk et al, 2010), and alcohol (Cippitelli et al, 2010; Zhao et al, 2012). A few of these studies implicated certain neural mechanisms within a circuit including the prefrontal cortex (Gonzalez et al, 2014; Kamei et al, 2005), hippocampus and entorhinal cortex (Zhao et al, 2012). However, none of these studies related the deficits in novel object recognition or the underlying substrates to relapse vulnerability. Our data suggest a cyclical pattern of meth use, cognitive deficits, and subsequent increased vulnerability to relapse. This cycle begins with a downregulation of perirhinal mGlu5 as a function of escalating meth use (Reichel et al, 2011), and restoring function at these receptors with an mGlu5 PAM rescued meth-induced deficits in novel object recognition in the present study. Relapse involving a choice between novel $v s$ meth-conditioned stimuli relies on similar mechanisms, perhaps owing to a common deficit in novelty salience. Thus, treatments aimed at restoring perirhinal mGlu5 signaling in meth addicts may be a viable therapeutic target for restoring novelty salience and reducing relapse.

\section{FUNDING AND DISCLOSURE}

This research was supported by NIH grants R01DA033049 and C06 RR015455. DPFE was a generous gift from the Vanderbilt Center for Neuroscience Drug Discovery. The authors declare no conflicts of interest.

\section{ACKNOWLEDGMENTS}

The authors thank Nastassia Bryant, Jennifer Hergatt, and Carole Berini for technical assistance, as well as the Vanderbilt Center for Neuroscience Drug Discovery for supplying DPFE.

\section{AUTHOR CONTRIBUTIONS}

All authors discussed the experimental design, results and implications, and commented on the manuscript. JP, MDS, CMR, and SMG contributed to and conducted the behavioral and surgical components. JP and JAH conducted the statistical analysis. JP wrote the initial manuscript. MDS, $\mathrm{JAH}$, and CMR gave technical support and conceptual advice, as well as reviewed and edited the manuscript.

\section{REFERENCES}

Ahmed SH, Koob GF (1998). Transition from moderate to excessive drug intake: change in hedonic set point. Science 282: 298-300.
Barker GR, Bashir ZI, Brown MW, Warburton EC (2006a). A temporally distinct role for group I and group II metabotropic glutamate receptors in object recognition memory. Learn Mem 13: $178-186$.

Barker GR, Warburton EC, Koder T, Dolman NP, More JC, Aggleton JP et al (2006b). The different effects on recognition memory of perirhinal kainate and NMDA glutamate receptor antagonism: implications for underlying plasticity mechanisms. J Neurosci 26: 3561-3566.

Bastle RM, Kufahl PR, Turk MN, Weber SM, Pentkowski NS, Thiel KJ et al (2012). Novel cues reinstate cocaine-seeking behavior and induce Fos protein expression as effectively as conditioned cues. Neuropsychopharmacology 37: 2109-2120.

Battaglia G, Fornai F, Busceti CL, Aloisi G, Cerrito F, De Blasi A et al (2002). Selective blockade of mGlu5 metabotropic glutamate receptors is protective against methamphetamine neurotoxicity. J Neurosci 22: 2135-2141.

Belcher AM, Feinstein EM, O'Dell SJ, Marshall JF (2007). Methamphetamine influences on recognition memory: comparison of escalating and single-day dosing regimens. Neuropsychopharmacology 33: 1453-1463.

Bevins RA, Besheer J (2005). Novelty reward as a measure of anhedonia. Neurosci Biobehav Rev 29: 707-714.

Briand LA, Gross JP, Robinson TE (2008). Impaired object recognition following prolonged withdrawal from extendedaccess cocaine self-administration. Neuroscience 155: 1-6.

Chesworth R, Brown RM, Kim JH, Lawrence AJ (2013). The metabotropic glutamate 5 receptor modulates extinction and reinstatement of methamphetamine-seeking in mice. PloS One 8: e68371.

Cippitelli A, Zook M, Bell L, Damadzic R, Eskay RL, Schwandt M et al (2010). Reversibility of object recognition but not spatial memory impairment following binge-like alcohol exposure in rats. Neurobiol Learn Mem 94: 538-546.

Dean AC, Groman SM, Morales AM, London ED (2013). An evaluation of the evidence that methamphetamine abuse causes cognitive decline in humans. Neuropsychopharmacology 38: 259-274.

Ennaceur A, Delacour J (1988). A new one-trial test for neurobiological studies of memory in rats. 1: Behavioral data. Behav Brain Res 31: 47-59.

Gass JT, Osborne MP, Watson NL, Brown JL, Olive MF (2008). mGluR5 antagonism attenuates methamphetamine reinforcement and prevents reinstatement of methamphetamine-seeking behavior in rats. Neuropsychopharmacology 34: 820-833.

Golembiowska K, Konieczny J, Wolfarth S, Ossowska K (2003). Neuroprotective action of MPEP, a selective mGluR5 antagonist, in methamphetamine-induced dopaminergic neurotoxicity is associated with a decrease in dopamine outflow and inhibition of hyperthermia in rats. Neuropharmacology 45: 484-492.

Gonzalez B, Raineri M, Cadet JL, Garcia-Rill E, Urbano FJ, Bisagno V (2014). Modafinil improves methamphetamineinduced object recognition deficits and restores prefrontal cortex ERK signaling in mice. Neuropharmacology 87: 188-197.

Gregory KJ, Herman EJ, Ramsey AJ, Hammond AS, Byun NE, Stauffer SR et al (2013). N-aryl piperazine metabotropic glutamate receptor 5 positive allosteric modulators possess efficacy in preclinical models of NMDA hypofunction and cognitive enhancement. J Pharmacol Exp Ther 347: 438-457.

Kamei H, Nagai T, Nakano H, Togan Y, Takayanagi M, Takahashi K et al (2005). Repeated methamphetamine treatment impairs recognition memory through a failure of novelty-induced ERK1/2 activation in the prefrontal cortex of mice. Biol Psychiatry 59: $75-84$.

Kealy J, Commins S (2011). The rat perirhinal cortex: a review of anatomy, physiology, plasticity, and function. Prog Neurobiol 93: 522-548. 
Kufahl PR, Hood LE, Nemirovsky NE, Barabas P, Halstengard C, Villa A et al (2012). Positive allosteric modulation of mGluR5 accelerates extinction learning but not relearning following methamphetamine self-administration. Front Pharmacol 3: 194.

Leventhal AM, Kahler CW, Ray LA, Stone K, Young D, Chelminski I, Zimmerman M (2008). Anhedonia and amotivation in psychiatric outpatients with fully remitted stimulant use disorder. Am J Addict 17: 218-223.

Malkova L, Forcelli PA, Wellman LL, Dybdal D, Dubach MF, Gale K (2015). Blockade of glutamatergic transmission in perirhinal cortex impairs object recognition memory in macaques. J Neurosci 35: 5043-5050.

Marshall JF, O'Dell SJ (2012). Methamphetamine influences on brain and behavior: unsafe at any speed? Trends Neurosci 35: 536-545.

Nawata Y, Kitaichi K, Yamamoto T (2012). Increases of CRF in the amygdala are responsible for reinstatement of methamphetamineseeking behavior induced by footshock. Pharmacol Biochem Behav 101: 297-302.

O'Dell SJ, Feinberg LM, Marshall JF (2010). A neurotoxic regimen of methamphetamine impairs novelty recognition as measured by a social odor-based task. Behav Brain Res 216: 396-401.

Olsen CM, Childs DS, Stanwood GD, Winder DG (2010). Operant sensation seeking requires metabotropic glutamate receptor 5 (mGluR5). PloS One 5: e15085.

Osborne MP, Olive MF (2008). A role for mGluR5 receptors in intravenous methamphetamine self-administration. Ann $N Y$ Acad Sci 1139: 206-211.

Parkitna JR, Sikora M, Golda S, Golembiowska K, Bystrowska B, Engblom D et al (2013). Novelty-seeking behaviors and the escalation of alcohol drinking after abstinence in mice are controlled by metabotropic glutamate receptor 5 on neurons expressing dopamine d1 receptors. Biol Psychiatry 73: 263-270.

Parmentier-Batteur S, O'Brien JA, Doran S, Nguyen SJ, Flick RB, Uslaner JM et al (2010). Differential effects of the mGluR5 positive allosteric modulator CDPPB in the cortex and striatum following repeated administration. Neuropharmacology 62: 1453-1460.

Pierce RC, Crawford CA, Nonneman AJ, Mattingly BA, Bardo MT (1990). Effect of forebrain dopamine depletion on noveltyinduced place preference behavior in rats. Pharmacol Biochem Behav 36: 321-325.

Rebec GV, Christensen JRC, Guerra C, Bardo MT (1997). Regional and temporal differences in real-time dopamine efflux in the nucleus accumbens during free-choice novelty. Brain Res 776: 61-67.

Reichel CM, Bevins RA (2008). Competition between the conditioned rewarding effects of cocaine and novelty. Behav Neurosci 122: $140-150$.

Reichel CM, Bevins RA (2010). Competition between novelty and cocaine conditioned reward is sensitive to drug dose and retention interval. Behav Neurosci 124: 141-151.

Reichel CM, Chan CH, Ghee SM, See RE (2012a). Sex differences in escalation of methamphetamine self-administration: cognitive and motivational consequences in rats. Psychopharmacology 223: 371-380.

Reichel CM, Gilstrap MG, Ramsey LA, See RE (2014). Modafinil restores methamphetamine induced object-in-place memory deficits in rats independent of glutamate $\mathrm{N}$-methyl-d-aspartate receptor expression. Drug Alcohol Depend 134: 115-122.

Reichel CM, Ramsey LA, Schwendt M, McGinty JF, See RE (2012b). Methamphetamine-induced changes in the object recognition memory circuit. Neuropharmacology 62: 1119-1126.

Reichel CM, Schwendt M, McGinty JF, Olive MF, See RE (2011). Loss of object recognition memory produced by extended access to methamphetamine self-administration is reversed by positive allosteric modulation of metabotropic glutamate receptor 5 . Neuropsychopharmacology 36: 782-792.

Reichel CM, See RE (2010). Modafinil effects on reinstatement of methamphetamine seeking in a rat model of relapse. Psychopharmacology 210: 337-346.

Rocha A, Kalivas PW (2010). Role of the prefrontal cortex and nucleus accumbens in reinstating methamphetamine seeking. Eur J Neurosci 31: 903-909.

Rogers JL, De Santis S, See RE (2008). Extended methamphetamine self-administration enhances reinstatement of drug seeking and impairs novel object recognition in rats. Psychopharmacology 199: 615-624.

Schenk S, Harper DN, Do J (2010). Novel object recognition memory: measurement issues and effects of MDMA selfadministration following short inter-trial intervals. J Psychopharmacol 25: 1043-1052.

Scofield MD, Trantham-Davidson H, Schwendt M, Leong KC, Peters J, See RE, Reichel CM (2015). Failure to recognize novelty after extended methamphetamine self-administration results from loss of long-term depression in the perirhinal cortex. Neuropsychopharmacology 40: 2526-2535.

Wang GJ, Volkow ND, Chang L, Miller E, Sedler M, Hitzemann R et al (2004). Partial recovery of brain metabolism in methamphetamine abusers after protracted abstinence. Am J Psychiatry 161: 242-248.

Warburton EC, Brown MW (2014). Neural circuitry for rat recognition memory. Behav Brain Res 285: 131-139.

Watterson LR, Kufahl PR, Nemirovsky NE, Sewalia K, Hood LE, Olive MF (2012). Attenuation of reinstatement of methamphetamine-, sucrose-, and food-seeking behavior in rats by fenobam, a metabotropic glutamate receptor 5 negative allosteric modulator. Psychopharmacology 225: 151-159.

Widholm JJ, Gass JT, Cleva RM, Olive MF (2011). The mGluR5 Positive Allosteric Modulator CDPPB Does Not Alter Extinction or Contextual Reinstatement of Methamphetamine-Seeking Behavior in Rats. Journal of addiction research \& therapy S1: pii 004.

Zhao YN, Wang F, Fan YX, Ping GF, Yang JY, Wu CF (2012). Activated microglia are implicated in cognitive deficits, neuronal death, and successful recovery following intermittent ethanol exposure. Behav Brain Res 236: 270-282.

Supplementary Information accompanies the paper on the Neuropsychopharmacology website (http://www.nature.com/npp) 